INSTITUT NATIONAL DE LA STATISTIQUE ET DES ETUDES ECONOMIQUES

Série des Documents de Travail du CREST

(Centre de Recherche en Economie et Statistique)

\title{
$n^{\circ}$ 2002-31
}

\section{La grenouille qui se peut faire aussi grosse que le boeuf}

\author{
P. FEVRIER \\ L. LINNEMER 2
}

Mars 2002

Les documents de travail ne reflètent pas la position de l'INSEE et n'engagent que leurs auteurs.

Working papers do not reflect the position of INSEE but only the views of the authors.

\footnotetext{
${ }^{1}$ INSEE et CREST-LEI-GRECSTA.

${ }^{2}$ CREST-LEI-GRECSTA et Université de Lille 2. Adresse : LEI-ENPC, 28 Rue des Saints-Pères, 75007 Paris, France. Email : linnemer@ensae.fr

Nous remercions vivement Anne Perrot, Romain Lesur et Michael Visser pour leurs remarques constructives.
} 


\title{
La grenouille qui se peut faire aussi grosse que le bouf
}

\author{
Philippe Février* et Laurent Linnemer ${ }^{\dagger \ddagger}$
}

Mars 2002

${ }^{*}$ INSEE et CREST-LEI-GRECSTA.

${ }^{\dagger}$ CREST-LEI-GRECSTA et Université de Lille 2, linnemer@ensae.fr. LEI-ENPC, 28, rue des Saints Pères, 75007 Paris, France.

${ }_{\ddagger}^{\ddagger}$ Nous remercions vivement Anne Perrot, Romain Lesur et Michael Visser pour leurs remarques constructives. 


\begin{abstract}
Quite often, a firm has to decide between investing in a safe project ("small") where rewards and losses remain moderate and a risky alternative ("big") where the rewards and the losses are larger. We study such a choice in a context where the probability of success depends both on the intrinsic ability of the firm and on the choice of an other firm which is a follower. The ability of the follower is unknown to the leader and vice-versa. We show that a leader with a poor ability selects the big project as well as a leader with a very high ability. On the other hand a leader with a medium ability prefers the small project. When weak the follower chooses the small project, when of medium ability it chooses randomly and when of high ability it selects the big project. Information asymmetry, though, prevents the selection of the best firm: a leader with a lower ability can deter competition form a medium or weak follower by selecting the big project.
\end{abstract}

Keywords: Entry deterrence, asymmetric information, bluff.

JEL Classification: C72, L1.

\title{
Résumé
}

Une entreprise fait souvent face au dilemme suivant: investir dans un "petit projet" où en cas de réussite le gain est modeste mais où les pertes restent modérées en cas d'échec, ou alors lancer un "grand projet" avec un gain plus important mais aussi des pertes plus conséquentes si le projet échoue. Nous étudions ce choix dans le cas où succès et échecs dépendent d'une part de l'habileté intrinsèque de l'entreprise et d'autre part du choix d'un concurrent (en position de suiveur) qui peut s'engager ou pas dans le "grand projet" et dont l'habileté est inconnue a priori. Un résultat surprenant émerge: une entreprise de type faible se lance dans le "grand projet" tout comme une entreprise de type fort tandis qu'une entreprise de type moyen préfère le "petit projet". La firme en position de suiveur sélectionne toujours le petit projet lorsqu'elle est de type faible, le grand projet si elle est d'un type élevé et choisit aléatoirement sinon. L'asymétrie d'information empêche dans ces circonstances la sélection du meilleur : un leader plus faible que son suiveur peut se retrouver seul sur le grand projet.

Mots clefs : Dissuasion de l'entrée, asymétrie d'information, bluff. JEL Classification : C72, L1. 


\section{Introduction}

Dans la fable «La grenouille qui se veut faire aussi grosse que le bœuf», Jean de La Fontaine décrit l'échec d'un vaniteux qui aurait aimé cacher sa véritable nature et susciter une admiration indue. Bien que La Fontaine ne s'intéresse qu'à la nature humaine dans la tradition des moralistes français, il semble que certaines entreprises sont dans la situation de la grenouille de la fable. En passant pour une firme qui produit de la meilleure qualité, ou qui a un meilleur bilan, ou un meilleur projet, une entreprise peut (en jouant sur des asymétries d'information) espérer accroître son profit. Toutefois, comme pour la grenouille, si la «tromperie» échoue, les conséquences peuvent être dramatiques. Nous développons un modèle où à l'équilibre une firme de faible qualité imite une firme de bonne qualité et réussit à décourager l'entrée d'un concurrent. Dans notre modèle la grenouille réussit à se faire passer pour un bœuf!

La sélection d'un nouveau projet dépend tout autant de la capacité à le mener à bien avec succès que de la présence éventuelle d'un concurrent. Dans un tel contexte et en présence d'asymétries d'information, le fait même de choisir un projet joue un rôle de signal qui peut décourager des concurrents potentiels. Nous nous intéressons au cas où une firme (dont la "force" est inconnue) peut, en choisissant la taille de son projet, écarter des concurrents. En fait, la simple décision de concourir pour un projet (plutôt que de ne pas le faire) signale l'habileté de l'entreprise.

Dans certains cas, ce problème prend une grande importance, en particulier, lorsque qu'il n'y a qu'un seul gagnant. Si plusieurs entreprises sont en concurrence sur un appel d'offres, par exemple, une seule l'emportera et les autres encourront des pertes. De même, si plusieurs firmes se lancent dans une course au brevet, seule la première à découvrir bénéficiera d'un profit de monopole tandis que les autres auront dépensé pour rien les frais de recherche. Même s'il est possible que deux entreprises se lancent en même temps dans un nouveau programme de recherche ou encore que deux firmes répondent en même temps à un appel d'offres, il est plus vraisemblable qu'un des deux acteurs prenne une telle décision avant l'autre. Dans un tel contexte (et si la décision de celui qui joue en premier n'est pas secrète) plusieurs questions émergent. Tout d'abord pour la firme qui décide en second: Faut-il participer à un appel d'offres sachant qu'un concurrent a déjà déposé une offre? Faut-il se lancer dans une course au brevet si une autre entreprise a déjà décidé de le faire? Mais aussi pour la firme en position de leader: Étant donné ses capacités à gagner la compétition le leader doit-il se lancer ou pas dans le projet?

Notre modèle fournit les réponses suivantes à ces questions : le leader doit toujours s'engager dans la compétition s'il est très faible ou très fort. S'il est de force intermédiaire il peut avoir intérêt à ne pas concourir. L'entreprise qui décide en second ne doit pas participer si elle est faible, toujours concourir si elle est très forte 
et elle peut adopter une stratégie mixte entre les deux. Si la stratégie de la firme en second n'est pas surprenante, celle du leader est plus spectaculaire notamment lorsqu'elle est très faible. À l'équilibre, il est optimal pour une firme très faible en position de leader de tenter un "coup de bluff" en se lançant dans la compétition.

Nous étudions en fait un modèle plus général où le choix du leader ne consiste pas simplement à décider entre participer ou pas à une compétition mais consiste à choisir la taille d'un projet ${ }^{1}$. Afin de garder un modèle simple tout en fournissant des intuitions intéressantes, nous nous limitons au cas où le leader peut choisir entre deux types de projets: un grand ou un petit ${ }^{2}$.

Le fait qu'une entreprise soit en position de leader peut s'expliquer de plusieurs manières. Par exemple, imaginons un laboratoire de recherche qui cherche à déposer un brevet. Toutefois elle doit choisir entre se lancer dans une course pour un brevet modeste ou alors ambitieux (récompense plus grande mais coûts plus importants en cas d'échec). Elle annonce publiquement son choix. Si elle choisit de viser le grand brevet, un laboratoire concurrent doit prendre la décision de se lancer dans la course ou pas. Appliqué à cette situation, notre modèle prédit qu'un laboratoire peu performant se lance toujours dans la course au brevet ambitieux et qu'il décourage parfois la concurrence. Cette intuition est certes obtenue dans un cadre très simple, mais elle s'étend au cadre plus traditionnel où la recherche aboutit de manière stochastique. Il existe de nombreux modèles de course aux brevets mais l'accent est souvent mis sur l'effort de R\&D comme dans Pérez-Castrillo and Verdier (1991) ou encore Bloch and Markowitz (1996) dans le cadre d'une recherche en plusieurs étapes.

L'autre situation bien illustrée par notre modèle est la suivante. Imaginons, une entreprise de conseil qui propose à une grande entreprise un projet d'étude. La grande entreprise met ce projet en concurrence en lançant un appel d'offres. Si le projet proposé est modeste, un concurrent apparaît automatiquement. L'idée étant qu'il existe forcément un concurrent avec un projet de ce type à soumettre. En revanche, le projet est ambitieux, la concurrence n'est que potentielle car le coût nécessaire à l'élaboration d'un grand projet est élevé. Un concurrent potentiel peut proposer un grand projet similaire mais s'il anticipe le projet déjà déposé est de qualité élevée tandis que son propre projet de qualité faible, il préfère renoncer. Cette tentation du concurrent à abandonner la compétition pour ne pas en subir les coûts pousse le leader à proposer un projet ambitieux alors même qu'il est de qualité basse.

Le bluff apparaît dans certains jeux. Le plus connu est le jeu de poker simplifié

1. Un projet peut consister à répondre à un appel d'offres plus ou moins complexe ou à se lancer dans une course au brevet dans un domaine plus ou moins difficile.

2. Remarquons que dans le cas limite où le petit projet ne rapporte et ne coûte rien nous sommes bien ramenés à un choix entre concourir ou pas. 
analysé par von Neumann and Morgenstern $(1944)^{3}$. Toutefois ce jeu décrit une situation très particulière et de plus il est à somme nulle. L'idée de relier les stratégies mixtes et la notion de bluff est ancienne comme le montre Sorin (1995). Dans cet article, Sorin présente aussi des modèles de réputation à la chain store paradox où certain type de bluff apparaît.

L'article est organisé de la manière suivante. La section 2 présente le modèle formel dont l'équilibre bayésien parfait est déterminé dans la section 3. Les propriétés de l'équilibre ainsi que d'autres interprétations sont discutées dans la section 4 et la section 5 conclut.

\section{Le modèle}

La (les) situation(s) économique(s) que nous avons à l'esprit peuvent être illustrées à l'aide du jeu suivant décrit schématiquement par la figure 1. La nature détermine le type $x$ de l'entreprise E1. La valeur de $x$ qui correspond à la «qualité» ou encore à «l'habileté» de E1 appartient à l'intervalle [0,1]. La valeur de $x$ est indépendante du projet choisi ${ }^{4}$. Si $x=0$, le projet de E1 (grand ou petit) est le plus mauvais possible. Au contraire, si $x=1$ le projet de E1 est le meilleur possible. L'entreprise E1 prend connaissance de $x$ et décide de "lancer" un gRand $(\mathrm{R})$ ou un Petit (P) projet. Si elle choisit un petit projet, alors la nature détermine $z \in[0,1]$ (la qualité du petit projet concurrent) selon une loi de probabilité uniforme. Le petit projet de E1 est un succès si et seulement si $x>z^{5}$. En cas de succès, E1 gagne $g \geq 0$ tandis que si son petit projet échoue, elle perd $c \geq 0$. Si l'entreprise E1 choisit un grand projet plutôt qu'un petit, alors la nature sélectionne $y \in[0,1]$ (la valeur du grand projet concurrent) selon une loi de probabilité uniforme. L'entreprise E2 apprend la valeur de $y$ et décide ou pas de concurrencer le grand projet de E1. Si elle décide de ne pas concurrencer E1 ("Dehors"), alors l'entreprise E1 réalise un gain G et E2 un gain (une perte) normalisé à zéro. En revanche, si E2 concurrence le projet de E1 ("Entre"), alors les gains sont les suivants. Si $x>y$ (resp. $x<y$ ), alors E1 gagne

3. Chapitre IV, section 19. Voir aussi Binmore (1992) chapitre 12.

4. Cette hypothèse peut sembler restrictive car on peut penser que pour réaliser un bon projet il faut une habileté supérieure. En fait, l'important est la capacité de E1 relative à celle de la concurrence. Imaginons par exemple que si $x$ est la qualité du petit projet, alors celle du grand projet est $a x+b$. Comme un petit projet n'est jamais comparé avec un grand, il n'est pas restrictif de supposer que $a=1$ et $b=0$.

5. Tout au long de l'article les événements du type $x=z$ qui arrivent avec une probabilité nulle sont ignorés. Typiquement, si $x=z$ l'entreprise E1 gagne $g$ avec une chance sur deux. 


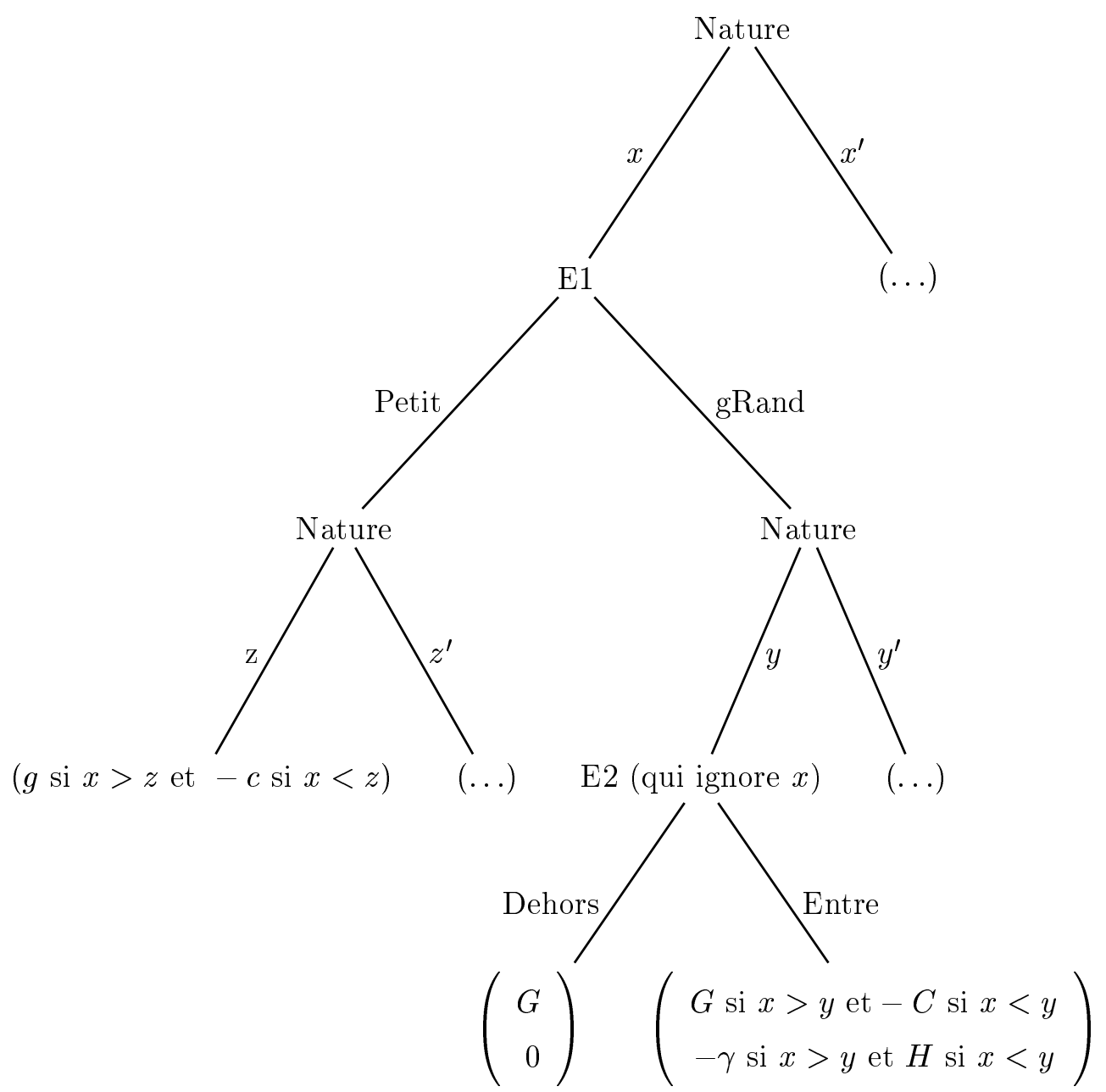

FIG. 1 - Choix d'un projet: arbre du jeu

$G$ (resp. E2 gagne $H$ ) et E2 perd $\gamma(\text { resp. E1 perd } C)^{6}$. Dans le cas particulier où $g=c=0$, la situation décrite est plus symétrique: E1 et E2 ont toutes les deux le choix d'entrer ou pas, mais E1 prend sa décision avant E2. Lorsque $c$ et $g$ ne sont pas nuls, la situation est un peu la même sauf que E1 possède une option de sortie plus ou moins attractive selon son type. Il aurait été possible d'offrir à E2 une option de sortie du même ordre que celle dont dispose E1. Cela n'aurait pas changé nos résultats. Nous avons préféré ne pas le faire pour garder l'accent sur la dimension "dissuasion de l'entrée". Il suffit que, pour E2, "entrer en concurrence" avec E1 soit plus coûteux

6. Si $x=y$, alors E1 gagne $G$ (resp. E2 gagne $H$ ) avec la probabilité $1 / 2$ et perd $C$ (resp. $\gamma$ ) avec la même probabilité. 
en cas d'échec que l'option "rester en dehors" pour que nos résultats restent valides. Une telle situation peut s'obtenir de différentes manières, la plus simple étant celle qui consiste à normaliser à 0 le gain de E2 s'il n'entre pas.

Il est important de noter que chaque projet, petit ou grand, est de la forme "le gagnant remporte tout". Par exemple, il peut s'agir d'une course au brevet: $1-x$ (resp. 1-y) mesure la rapidité avec laquelle E1 (resp. E2) met au point son prototype et dépose un brevet. La première entreprise à déposer un brevet acquière un pouvoir de monopole sur son exploitation. Bien entendu de la même manière, dans le cas d'un appel d'offres, seule l'entreprise sélectionnée a un gain.

Par définition, un petit projet est moins rentable qu'un grand projet en cas de succès: $g<G$. Toutefois l'échec est plus cuisant avec un grand plutôt qu'un petit projet: $c<C$. Pour l'entreprise E2, il est supposé que $H>0$ et que $\gamma>0$. En revanche, il n'est pas fait d'hypothèse particulière sur la position de $H$ vis-à-vis de $G$ ni de $C$ par rapport à $\gamma$.

Il est important de commencer par définir la notion de rentabilité de chaque projet et c'est l'objet du lemme 1.

\section{Lemma 1.}

Il existe $\widetilde{x} \in[0,1]$ tel que pour tout $x$ inférieur (resp. supérieur) à $\widetilde{x}$, l'espérance de gain du petit projet est supérieure (resp. inférieure) à celle d'un grand projet concurrencé.

Il existe $\widetilde{y} \in[0,1]$ tel que pour tout y inférieur (resp. supérieur) à $\widetilde{y}$, l'espérance de gain liée à l'entrée, sachant que E1 choisit le grand projet quel que soit $x$, est négative (resp. positive).

Démonstration. Notons $E_{P}=-(1-x) c+x g$ l'espérance de gain du petit projet pour une entreprise E1 de type $x$. De la même manière, soit $E_{R}=-(1-x) C+x G$ l'espérance de gain du grand projet pour E1 lorsque E2 entre toujours. Il est facile de vérifier que

$$
E_{P} \geq E_{R} \Leftrightarrow x \leq \widetilde{x}=\frac{C-c}{C-c+G-g}
$$

De la même manière, notons $E_{E}=-(1-y) \gamma+y H$ l'espérance de gain de E2 qui choisit d'entrer lorsque E1 choisit le grand projet quel que soit $x$. Il est immédiat que

$$
E_{E} \leq 0 \Leftrightarrow y \leq \widetilde{y}=\frac{\gamma}{\gamma+H}
$$




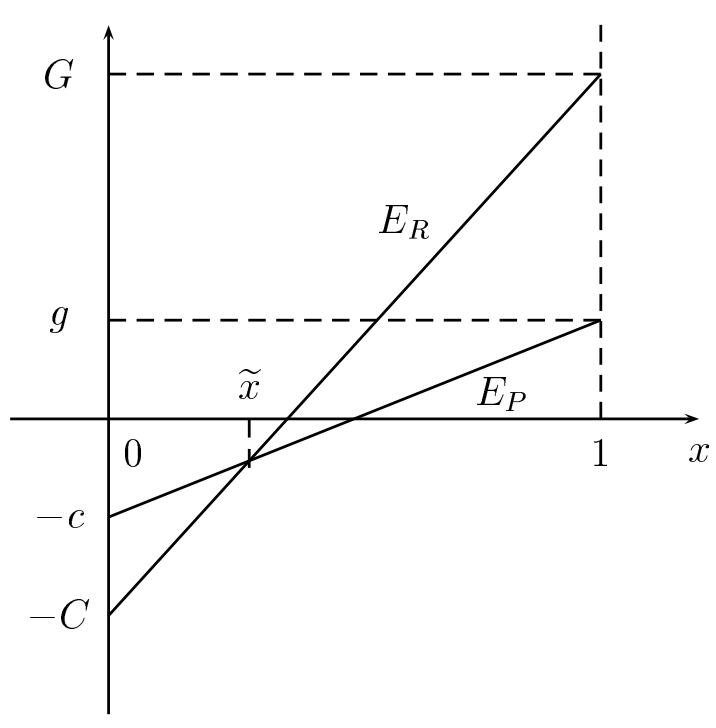

FIG. 2 - Espérances de gains pour E1 FIG. 3 - Espérance de gain pour E2 lorsque lorsque E2 entre toujours

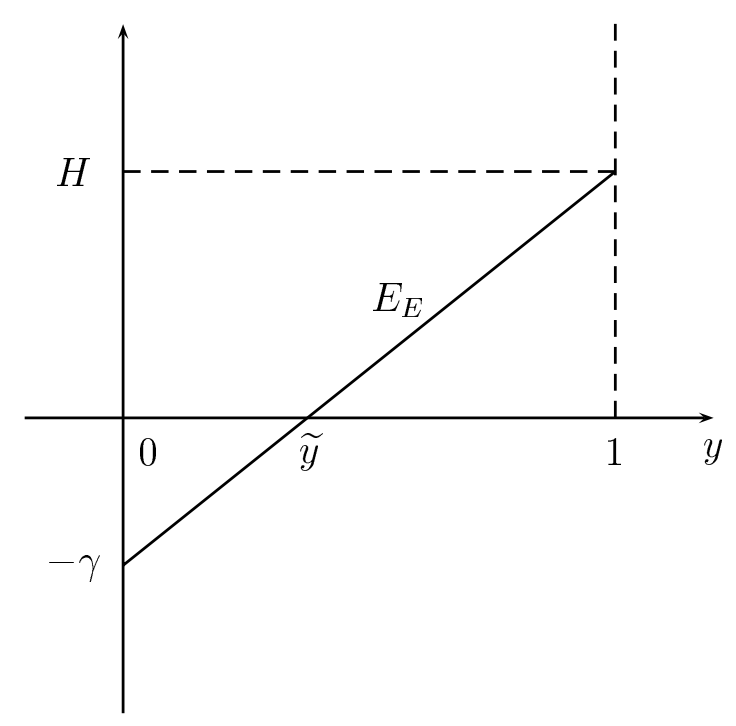

E1 choisit toujours le grand projet

La figure 2 montre comment varient les espérances de gain de E1 en fonction de $x$. Clairement, si $x=0$ le petit projet a une meilleure espérance de gain que le grand (il limite les pertes). En revanche, si $x=1$ le grand projet domine le petit. Comme les deux espérances sont des fonctions (affines) croissantes avec $x$, il existe une probabilité seuil $\widetilde{x}$, telle qu'en dessous le petit projet est préféré et inversement au dessus. La figure 3 est similaire pour l'entreprise E2, la comparaison se faisant entre 0 et l'espérance de gain $E_{E}$ en cas d'entrée.

Les valeurs seuils $\widetilde{x}$ et $\widetilde{y}$ varient de manière intuitive avec les paramètres. Si $C$ tend vers $c$, alors $\widetilde{x}$ tend vers 0 . En effet, si la perte en cas d'échec est la même pour les deux projets, il est toujours préférable de choisir le grand projet qui rapporte plus en cas de succès. En revanche, si $G$ tend vers $g, \widetilde{x}$ tend vers 1: le grand projet est toujours moins avantageux que le petit (même gains mais pertes plus grandes).

\section{3 Équilibre}

Puisqu'il s'agit d'un jeu séquentiel, nous concentrons notre attention sur la détermination des équilibres bayésiens parfaits. Un équilibre bayésien parfait consiste 
d'une part en une stratégie comportementale $\sigma_{1}($.$) pour l'entreprise E1, c'est-à-dire$ une fonction qui à tout $x$ associe la probabilité $\sigma_{1}(x)$ avec laquelle l'entreprise E1 de type $x$ choisit le grand projet; d'autre part, en une stratégie comportementale $\sigma_{2}($.$) pour l'entreprise E2, c'est-à-dire une fonction qui à tout y$ associe la probabilité $\sigma_{2}(y)$ avec laquelle l'entreprise E2 de type $y$ choisit "Entre" sachant que E1 a sélectionné le grand projet. Par définition de l'équilibre, $\sigma_{1}$ et $\sigma_{2}$ doivent être des meilleures réponses l'une à l'autre.

Nous pouvons distinguer trois grands cas a priori. Tout d'abord celui où l'entreprise E1 choisit le grand projet quel que soit son type. Ensuite le cas où seuls les types élevés choisissent le grand projet tandis que les types faibles sélectionnent le petit. Enfin, le cas (le plus surprenant) dans lequel les types les plus faibles comme les plus forts se lancent dans le projet le plus ambitieux tandis que les types intermédiaires choisissent le petit projet. Ces trois situations vont être étudiées successivement. Nous commençons toutefois par résoudre le jeu en information parfaite afin de fournir un point de repère.

\subsection{Information parfaite}

Dans l'étude d'un jeu à information asymétrique, il est souvent utile de décrire quelle en serait l'issue si l'information était plus symétrique. Ici, nous pouvons supprimer l'asymétrie d'information à deux niveaux: tout d'abord nous envisageons le cas où E1 (resp. E2) connaît le type de E2 (resp. E1) avant de prendre sa décision. Nous supposons ensuite que E1 doit faire son choix dans l'ignorance du type de E2, tandis que E2 entre ou pas après avoir observé le type de E1. La firme E2 dispose donc d'un avantage informationnel qui compense en quelque sorte sa position de suiveur.

Si E1 connaît le type de E2 avant de sélectionner un projet et sait que E2 connaît son type, son choix est particulièrement simple. Si $x>y$, il choisit le grand projet et E2 décide de ne pas entrer. En revanche, si $x<y$, E1 choisit le petit projet et s'il choisissait le grand, alors E2 déciderait d'entrer. En effet, puisque la compétition est coûteuse tandis que son issue est parfaitement prévisible, elle n'a jamais lieu.

La situation où E1 prend sa décision en ignorant le type de E2 mais en sachant que E2 connaîtra le sien avant de décider d'entrer ou pas conduit à un résultat plus intéressant. Du point de vue de E1, ce cas de figure est identique à celui où E2 entre toujours. En effet, E2 entre si et seulement si $x<y$ et gagne la compétition. S'il entrait quel que soit $y$, l'espérance de gain de E1 serait identique (il perdrait effectivement si $x<y$ et il gagnerait si $x>y$ ) la seule différence est que lorsque E1 gagne, E2 n'a pas de coût. Étant donnée la stratégie de E2, E1 choisit le grand projet si $x>\widetilde{x}$ et sélectionne le petit si $x<\widetilde{x}$. Ce résultat correspond à l'intuition: 
si E1 est fort, il n'a pas peur de la compétition et il se lance dans le grand projet et inversement s'il est faible.

\subsection{Tout le monde voit en grand}

La proposition 1 précise pour quelles valeurs des paramètres il existe un équilibre bayésien parfait où l'entreprise E1 choisit le grand projet quel que soit son type. La proposition 3 montre de plus que pour ces valeurs des paramètres il s'agit de l'unique équilibre bayésien parfait.

Proposition 1. Si $\widetilde{y} \geq \widetilde{x}$, alors il existe un unique équilibre bayésien parfait caractérisé par :

$$
\forall x \in[0,1], \sigma_{1}^{*}(x)=1 \text { et } \sigma_{2}^{*}(y)= \begin{cases}0 & \text { si } y<\widetilde{y} \\ 1 & \text { si } y \geq \widetilde{y}\end{cases}
$$

Démonstration. Supposons tout d'abord que pour tout $x, x \in[0,1], \sigma_{1}^{*}(x)=1$. L'espérance de gain d'une entreprise E2 de type $y$ qui joue E s'écrit:

$$
\pi_{2}\left(y, E, \sigma_{1}^{*}\right)=H \int_{0}^{y} d x-\gamma \int_{y}^{1} d x=-\gamma+(\gamma+H) y
$$

L'entreprise E2 joue donc E si et seulement si cela lui assure une espérance de gain supérieure ou égale à zéro soit si $y \geq \widetilde{y}=\frac{\gamma}{\gamma+H}$.

Supposons maintenant que la stratégie de l'entreprise E2 de type $y$ consiste à rester dehors si $y<\widetilde{y}$ et à entrer si $y \geq \widetilde{y}$. L'espérance de profit d'une entreprise E1 de type $x$ qui choisit le grand projet s'écrit

$$
\pi_{1}\left(x, R, \sigma_{2}^{*}\right)=\left\{\begin{aligned}
& G \int_{0}^{\widetilde{y}} d y-C \int_{\widetilde{y}}^{1} d y=-C+(C+G) \widetilde{y} \text { si } x<\widetilde{y} \\
& G \int_{0}^{\widetilde{y}} d y+G \int_{\widetilde{y}}^{x} d y-C \int_{x}^{1} d y=-C+(C+G) x \quad \text { si } x \geq \widetilde{y}
\end{aligned}\right.
$$

tandis que son espérance de profit liée au petit projet est

$$
\pi_{1}(x, P)=\int_{0}^{x} g d z-\int_{x}^{1} c d z=(g+c) x-c
$$

Il est immédiat de vérifier que si $\pi_{1}\left(\widetilde{y}, R, \sigma_{2}^{*}\right) \geq \pi_{1}(\widetilde{y}, P)$, alors pour tout $x, \pi_{1}\left(x, R, \sigma_{2}^{*}\right) \geq$ $\pi_{1}(x, P)$. Or, (sous réserve que $G>g$ et $H>0$ )

$$
\pi_{1}\left(\widetilde{y}, R, \sigma_{2}^{*}\right) \geq \pi_{1}(\widetilde{y}, P) \Leftrightarrow \frac{\gamma}{H} \geq \frac{C-c}{G-g} \Leftrightarrow \widetilde{y} \geq \widetilde{x}
$$


L’unicité est prouvée avec la démonstration de la proposition 3.

La figure 4 représente l'espérance de gain de E1 lorsque E2 joue $\sigma_{2}^{*} \cdot{ }^{7}$ Cet équilibre

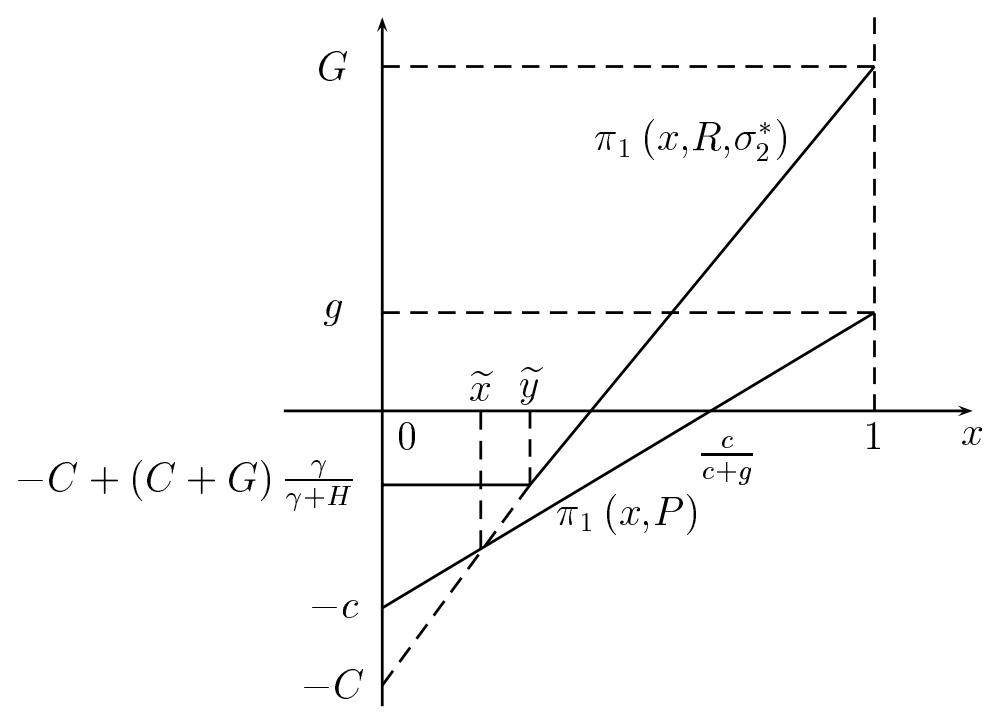

FIG. 4 - Espérances de gains à l'équilibre

émerge dans le cas où en moyenne la rentabilité du grand projet (en concurrence) est plus importante pour E1 que pour E2, c'est-à-dire lorsque $\widetilde{y}>\widetilde{x}$. Puisque E1 choisit toujours le grand projet, E2 n'entre (par définition de $\widetilde{y}$ ) que si $y \geq \widetilde{y}$. Il en résulte que pour tout $x \leq \widetilde{y}$ l'espérance de gain de E1 est constante: elle gagne $G$ avec la probabilité $\widetilde{y}$ et perd $C$ avec la probabilité $1-\widetilde{y}$. En revanche, pour $x \geq \widetilde{y}$ l'espérance de gain dépend de $x$ et il s'agit exactement de l'espérance de gain de E1 lorsque E2 entre toujours. En effet, si $y<x$ l'espérance de gain de E1 n'est pas affectée par la décision de E2. Il en découle que le grand projet est préférable au petit si et seulement si $x \geq \widetilde{x}$ ce qui est bien le cas puisque $\widetilde{y}>\widetilde{x}$.

\subsection{Les bons d'un côté les mauvais de l'autre}

Dans cette section, nous montrons qu'il n'existe pas d'équilibre où E1 choisit le grand projet si et seulement si son type $x$ est supérieur à un certain seuil. C'est-à-dire que l'équilibre qui prévaut lorsque E1 ignore le type de E2 mais E2 connaît le type de E1, disparaît lorsque E2 ignore le type de E1. En information (réciproquement)

7. Notons que sur cette figure $\frac{\gamma}{\gamma+H}$ est inférieur à $\frac{c}{c+g}$ et donc $-C+(C+G) \frac{\gamma}{\gamma+H}$ est négatif. Si $\frac{\gamma}{\gamma+H}$ était supérieur à $\frac{c}{c+g}$, alors $-C+(C+G) \frac{\gamma}{\gamma+H}$ serait positif. 
asymétrique, il n'existe pas d'équilibre où les mauvais types sélectionnent le petit projet tandis que les types élevés optent pour le grand projet. La proposition 2 précise ce point.

Proposition 2. Il n'existe pas d'équilibre bayésien parfait du type

$$
\begin{gathered}
\sigma_{1}^{*}(x)= \begin{cases}0 & \text { si } x<\widehat{x} \\
1 & \text { si } x \geq \widehat{x}\end{cases} \\
\text { avec } \widehat{x}>0
\end{gathered}
$$

Démonstration. Si E1 joue une stratégie à seuil, $\widehat{x}$, alors l'espérance de gain de E2 s'écrit s'il entre

$$
\pi_{2}\left(y, E, \sigma_{1}^{*}\right)=\left\{\begin{array}{cl}
-\gamma & \text { si } y \leq \widehat{x} \\
H \int_{\widehat{x}}^{y} \frac{d x}{1-\widehat{x}}-\gamma \int_{y}^{1} \frac{d x}{1-\widehat{x}} & \text { si } y \geq \widehat{x}
\end{array}\right.
$$

et donc (il est facile de vérifier que) la meilleure réponse de E2 est

$$
\sigma_{2}^{*}(y)= \begin{cases}0 & \text { si } y<\widehat{y} \\ 1 & \text { si } y \geq \widehat{y}\end{cases}
$$

avec

$$
\widehat{y}=\frac{\gamma+\widehat{x} H}{\gamma+H}>\widehat{x}
$$

ce qui signifie que E2 n'entre jamais pour $y<\widehat{y}$. Mais alors, l'espérance de gain liée au grand projet est constante pour tout $x \leq \widehat{y}$, et comme en $\widehat{y}$ le grand projet est préféré puisque $\widehat{y}>\widehat{x}$, c'est que le grand projet est préféré pour tout $x$. Nous sommes donc ramené à la proposition 1 .

Ce résultat négatif, souligne que d'une certaine manière, il n'existe pas d'équilibre "révélateur" où le choix «grand projet» signale un type fort tandis que le choix «petit projet» révèle un type faible. À l'équilibre certains types faibles doivent «imiter»le comportement des types forts. 


\subsection{De l'audace!}

Dans cette section, nous étudions en détail le cas où les types les plus faibles comme les plus forts se lancent dans le projet le plus ambitieux tandis que les types intermédiaires choisissent le petit projet. À un tel équilibre, l'entreprise E1 de type faible joue sur un «bluff». Elle s'engage de manière audacieuse dans le grand projet en anticipant que l'entreprise E2 restera dehors avec une probabilité suffisamment élevée. La proposition 3 établit qu'il n'existe qu'un seul équilibre bayésien parfait. Plus exactement, il n'existe qu'une seule famille d'équilibres bayésiens parfaits.

Proposition 3. Si $\widetilde{x} \geq \widetilde{y}$, alors les stratégies d'équilibres ont la forme suivante:

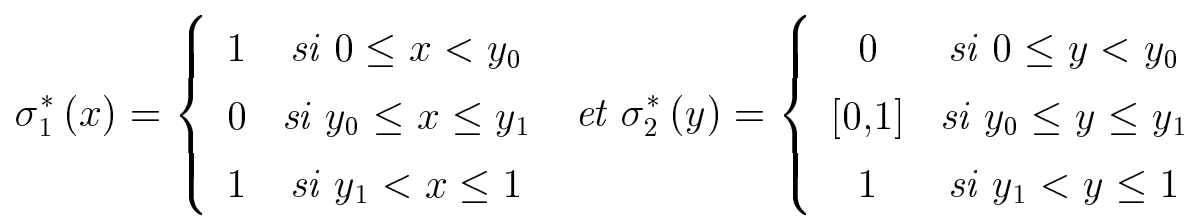

avec

$$
\begin{aligned}
y_{0} & =\frac{\widetilde{y}}{1-\widetilde{y}}(1-\widetilde{x})=\frac{\gamma}{H} \frac{G-g}{(G-g)+(C-c)} \\
y_{1} & =\widetilde{x}=\frac{C-c}{(G-g)+(C-c)} \\
\sigma_{2}^{*} \text { quelconque telle que } \int_{y_{0}}^{y_{1}} \sigma_{2}^{*}(y) d y & =\left(\frac{g+G}{c+C}\right) \frac{H(C-c)-\gamma(G-g)}{H((G-g)+(C-c))}
\end{aligned}
$$

Démonstration. Voir l'annexe A

Les figures 5 et 6 montrent les espérances de gains à l'équilibre de la proposition 3 pour chacun des joueurs et permettent de donner l'intuition du résultat.

Tout d'abord, le comportement de E2 est assez facile à comprendre lorsque $y$ est proche de 0 ou de 1 . Si $y$ est proche de zéro, il n'entre pas puisqu'en moyenne E1 sera toujours plus fort que lui. En revanche si $y$ est proche de 1, E2 tente sa chance. Puisque E2 n'entre jamais si $y<y_{0}$, l'espérance de gain de E1 est constante pour tout $x<y_{0}$. Et puisque E2 entre toujours si $y>y_{1}$, l'espérance de gain de E1 lorsque $x>y_{1}$ correspond exactement à l'espérance de E1 lorsque E2 entre toujours. Or, (voir lemme 1) face à un adversaire toujours présent, E1 préfère le grand projet si et seulement si $x>\widetilde{x}$. Il est donc cohérent de trouver $y_{1}=\widetilde{x}$. Lorsque $x$ est petit, nous avons vu que l'espérance de gain liée au grand projet était constante. Comme justement à l'équilibre E2 n'entre pas trop souvent, un type $x=0$ préfère le grand projet au petit. Toutefois, l'espérance de gain liée au petit projet est croissante avec 


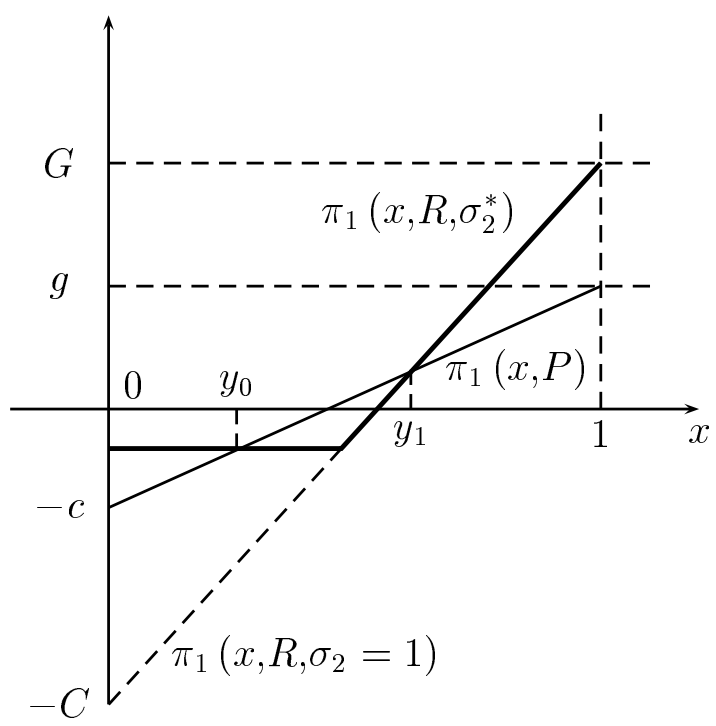

FIG. 5 - Espérances de gains pour E1 à FIG. 6 - Espérances de gains pour E2 à l'équilibre avec bluff l'équilibre avec bluff

$x$, il arrive un point $\left(x=y_{0}\right)$ où le petit projet devient plus intéressant que le grand et donc une entreprise E1 de type intermédiaire préfère choisir le petit projet (sinon nous serions dans le cas de la proposition 1).

La partie la moins intuitive du résultat est le comportement de E2 entre $y_{0}$ et $y_{1}$. Quel que soit $y_{0}<y<y_{1}$, une entreprise E2 de type $y$ sait qu'elle n'aura pas d'adversaire "équivalent". Soit elle a un adversaire plus faible $x<y_{0}$ soit un adversaire plus fort $x>y_{1}$. Donc quel que soit $y_{0}<y<y_{1}$, une entreprise E2 de type $y$ prend la même décision. Or, il est facile de voir qu'il est impossible que pour ces types E2 entre toujours ou n'entre jamais. En effet, si elle entrait toujours, une entreprise E1 de type $x=y_{0}$ ne serait indifférente entre le grand et le petit projet que si $y_{0}=\widetilde{x}$, mais alors $y_{0}=y_{1}$ c'est-à-dire que E1 entrerait toujours (proposition 1) ce qui n'est pas possible ici. Si E2 n'entre jamais pour $y_{0}<y<y_{1}$, cela veut dire que E2 n'entre jamais pour $0<y<y_{1}$, et il est donc impossible que E1 ne choisisse pas le grand projet pour $y_{0}<x<y_{1}$. Il en résulte que E2 doit jouer en stratégie mixte pour $y_{0}<y<y_{1}$, et cette stratégie mixte doit être telle qu'elle laisse une entreprise E1 de type $y_{0}$ indifférente entre le grand et le petit projet. Cette condition ne définit pas une unique stratégie comportementale $\sigma_{2}^{*}(y)$ pour $y_{0}<y<y_{1}$. Sur la figure 5, il a été supposé que E2 jouait selon une stratégie à seuil: ne pas entrer si $y_{0}<y<\widehat{y}$, et entrer si $\widehat{y}<y<y_{1}$ (avec $\widehat{y}$ bien choisi). Toutefois comme la seule chose qui compte est la valeur de l'intégrale $I=\int_{y_{0}}^{y_{1}} \sigma_{2}^{*}(y) d y$, d'autres (une infinité d'autres) 
fonctions $\sigma_{2}^{*}$ conviendraient. Sur la figure 5 cela changerait la forme de l'espérance de gain de E1 pour $y_{0}<x<y_{1}$. Au lieu d'avoir une droite horizontale puis une droite croissante, on aurait dans un cadre plus général une fonction croissante qui partirait du point $\left(y_{0}, G-(C+G) I\right)$ et qui arriverait au point $\left(y_{1}, G y_{1}-C\left(1-y_{1}\right)\right)$ mais qui resterait toujours inférieure à la droite $\pi_{1}(x, P)$.

\section{Discussion}

Dans cette section, nous discutons tout d'abord les propriétés de l'équilibre. Nous étudions en particulier l'émergence de tel ou tel type d'équilibre en fonction des valeurs des paramètres. Nous donnons ensuite d'autres interprétations possibles au modèle.

\subsection{Propriétés de l'équilibre}

La première propriété frappante de notre modèle est que quel que soit l'équilibre qui apparaît, l'entreprise E1 de type le plus faible se lance toujours dans le grand projet. En effet, si $\widetilde{x}<\widetilde{y}$, l'équilibre est celui de la proposition 1 et E1 choisit le grand projet quel que soit son type. Ou alors, si $\widetilde{x}>\widetilde{y}$, l'équilibre est celui de la proposition 3 et E1 choisit le grand projet pour $x<y_{0}$ et donc en particulier si $x=0$. Ce résultat, à première vue contre intuitif, s'explique bien: si E1 est de type $x=0$, elle est sure de perdre $-c$ en choisissant le petit projet. En revanche en sélectionnant le grand projet, elle bénéficie du fait qu'en espérance E2 va abandonner la compétition.

Les figures 7 et 8 montrent quel équilibre prévaut en fonction des valeurs des paramètres. Tout d'abord la figure 7 représente $y_{0}$ et $y_{1}$ en fonction de $\widetilde{x}$. La valeur seuil $y_{1}$ est une droite croissante avec $\widetilde{x}$, tandis que $y_{0}$ est une droite décroissante, dont la pente et l'ordonnée à l'origine dépendent du paramètre $\widetilde{y}$. Plus précisément elles sont égales à $\frac{\widetilde{y}}{1-\widetilde{y}}=\frac{\gamma}{H}$. Toutefois, il n'est pas forcément très facile d'interpréter le paramètre $\widetilde{x}$. Pour contourner cette difficulté, il est possible de représenter, comme dans la figure 8, $y_{0}$ et $y_{1}$ comme des fonctions de $\tau_{1}=\frac{\widetilde{x}}{1-\widetilde{x}}=\frac{C-c}{G-g}$. Le rapport $\tau_{1}$ (entre l'accroissement des pertes et celui des gains lorsque E1 passe du petit au grand projet) mesure l'accroissement du risque qu'il y a à choisir le grand projet plutôt que le petit. Par exemple, si $C=c$ et $G>g$, il est clair que le grand projet domine le petit et dans ce cas le rapport est nul traduisant l'idée que le grand projet ne présente aucun risque par rapport au petit. En revanche si $C>c$ tandis que $G=g$, alors c'est le petit projet qui domine le grand, et le rapport est infini. Il est important de 

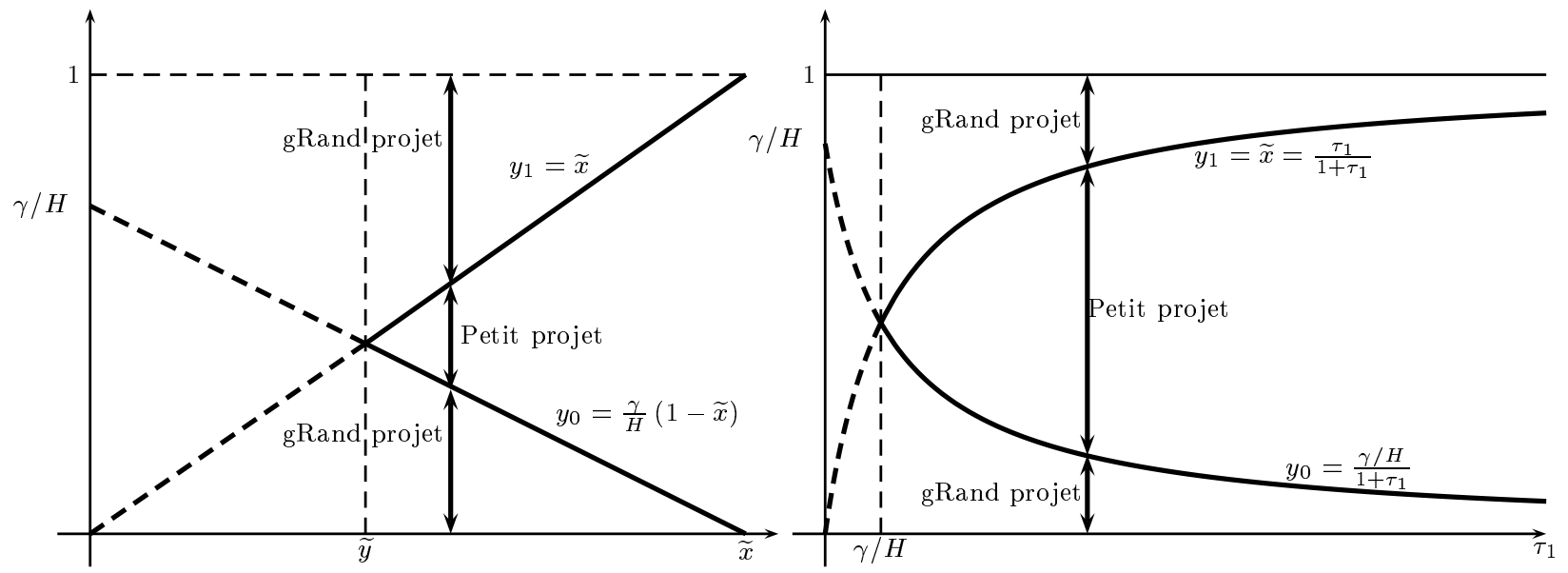

FIG. 7 - Variation de $y_{0}$ et $y_{1}$ en fonction FIG. 8 -Variation de $y_{0}$ et $y_{1}$ en fonction de $\widetilde{x}$

$$
\text { de } \tau_{1}=\frac{C-c}{G-g}
$$

remarquer que $\gamma / H>\tau_{1} \Leftrightarrow \widetilde{y}>\widetilde{x}$. La figure 8 montre que lorsque $\tau_{1}$ est inférieur à $\gamma / H$, alors le grand projet est sélectionné quel que soit $x$. En revanche lorsque $\tau_{1}$ dépasse $\gamma / H$, le grand projet n'est plus choisi que par les types faibles $x<y_{0}$ et forts $x>y_{1}$. Si $\tau_{1}$ tend vers l'infini, alors $y_{0}$ tend vers 0 et $y_{1}$ tend vers 1 .

Si E1 choisi le grand projet, et lorsque $\tau_{1}>\frac{\gamma}{H}$, alors son type est en moyenne égal à

$$
E[x \mid R]=\frac{\left(\frac{\gamma}{H}\right)^{2}+1+2 \tau_{1}}{2\left(1+\frac{\gamma}{H}\right)\left(1+\tau_{1}\right)}
$$

Il est facile de vérifier que $E[x \mid R]>1 / 2$ si et seulement si $H>\gamma$. Il en résulte que si $\gamma>H$, alors une entreprise E1 qui choisit le grand projet est en moyenne moins forte qu'une entreprise E1 qui choisit le petit projet. De plus, $E[x \mid R]$ est une fonction strictement croissante de $\tau_{1}$ si $\gamma<H$ et une fonction strictement décroissante si $\gamma>H$.

La figure 9 présente l'issue du jeu en fonction de la valeur du type de E1 et de celui de E2 (ou en fonction de $z$ si E1 choisit le petit projet). La figure illustre le cas où $\widetilde{x}>\widetilde{y}$.

Lorsque $x<y_{0}$, E1 choisit le grand projet, il s'agit d'une certaine manière d'un bluff puisque (à l'équilibre) cette stratégie n'est payante que si E2 n'entre pas. Si 
$y<y_{0}$, E2 évite la compétition est donc le bluff est réussi. En revanche, si $y>y_{1}$, E2 entre et il gagne. Dans ce cas le bluff est puni. Enfin, si $y_{0}<y<y_{1}$, E2 entre avec la probabilité $\sigma_{2}^{*}(y)$ et donc le bluff réussi ou échoue selon que E2 entre ou pas.

Pour $y_{0}<x<y_{1}$, l'entreprise E1 préfère sélectionner le petit projet. Ce choix conduit à un gain si $z<x$ et à une perte si $z>x$ d'où la droite en pointillé.

Enfin, si $y_{1}<x$, l'entreprise E1 se décide pour le grand projet. Comme son type est plutôt élevé, il ne s'agit pas d'un bluff mais d'une volonté de profiter d'un $x$ grand pour gagner la compétition. Cette stratégie est couronnée de succès tant que $y<y_{1}$ (que E2 décide alors d'entrer ou pas) tandis qu'elle peut conduire à un échec si $y>x>y_{1}$.

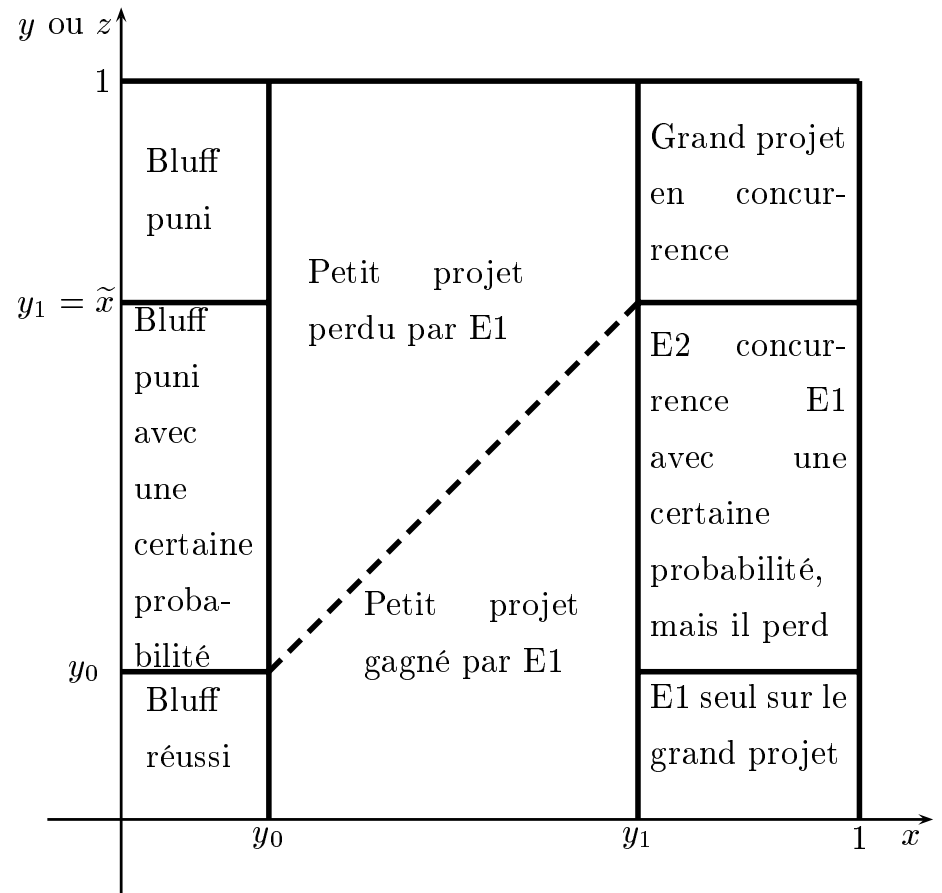

FIG. 9 - Issue du jeu selon $x$ et $y$ 


\subsection{Autres interprétations}

Un situation que notre modèle peut illustrer de manière amusante est celle des étudiants qui doivent rendre un mémoire ${ }^{8}$. L'étudiant (ou le groupe d'étudiants) a une capacité $x$ à réaliser un mémoire. Il n'est pas directement en concurrence avec les autres étudiants mais le(s) correcteur(s) juge(nt) avec un niveau de sévérité $y$ le niveau du mémoire. Les étudiants ont clairement un coût à réaliser un mémoire et celui-ci est plus important pour le mémoire ambitieux. Pour simplifier, on peut supposer que les notes varient de la manière suivante : un petit (resp. grand) mémoire qui passe les exigences du jury obtient 12 (resp. 18) et sinon 8 (resp. 8). La lecture d'un petit mémoire n'entraîne aucun coût pour le jury. En revanche, la lecture/évaluation d'un mémoire ambitieux est coûteuse. Le jury peut donc être tenté de lire de manière superficielle un grand mémoire et lui donner une bonne note (c'est-à-dire "ne pas entrer" dans l'évaluation).

À l'équilibre notre modèle indique que les étudiants se répartissent en trois groupes: celui des bons (ou très bons) étudiants qui choisissent de faire un mémoire ambitieux, le groupe des étudiants moyens qui optent pour le petit mémoire et enfin le groupe des mauvais (très mauvais) étudiants qui se lancent dans un projet ambitieux. Leur mémoire n'est en fait que de la poudre aux yeux: de beaux graphiques et un texte bien écrit mais copiés dans un ou plusieurs livres. Toutefois s'ils tombent sur un jury négligeant, ils ont 18 alors qu'avec un petit projet ils avaient plus de chance de se faire épingler (et d'obtenir 8). Le jury s'adapte à cette stratégie de la manière suivante: un jury naturellement négligent «fait confiance» aux étudiants et ne contrôle pas la qualité des mémoires difficiles. Un jury de capacité intermédiaire joue en stratégie mixte et contrôle ou pas avec une certaine probabilité. Enfin, un jury sévère contrôle avec certitude.

Cette exemple montre que la présence d'un concurrent n'est pas nécessaire pour faire fonctionner notre modèle. L'entreprise E2 (ou encore un gouvernement) peut être le commanditaire du projet plutôt qu'un concurrent. La firme E1 propose un petit projet ou un grand projet. S'il s'agit d'un petit projet, sa qualité est toujours évaluée et il est accepté que si elle dépasse les exigences de E2 $(x>y)$. En revanche, s'il s'agit d'un grand projet, la direction est enthousiaste (préférence des dirigeants pour les grands projets (white elephants)) et souhaite le mettre en œuvre (sans vérifier si $x>y$ ou pas). Le contrôleur financier (par exemple) doit donner son avis. S'il impose une expertise coûteuse à l'entreprise, il se place dans une situation

8. Le petit projet correspond à un travail honnête mais d'ambition modeste (disons un mémoire d'une trentaine de page basé sur des lectures sérieuses et des commentaires personnels). Le grand projet correspond à un mémoire ambitieux (de cinquante à cent pages, avec des graphiques, des photos, des listings informatiques en annexes, etc....). 
délicate car il retarde la mise en route du projet. S'il s'avère que le projet était de bonne qualité, il est donc pénalisé $(-\gamma<0)$ en revanche si le contrôle révèle que E1 proposait un projet de mauvaise qualité la direction le récompense $(H>0)$. Notre modèle montre qu'à l'équilibre des grands projets de mauvaise qualité seront mis en œuvre malgré la présence (potentielle) d'un contrôle.

Comme nous invite à le penser la fable de La Fontaine notre modèle s'applique à la vie des animaux. En effet, le signal est un thème fréquemment étudié en biologie depuis le travail novateur de Zahavi $(1975)^{9}$. Son analyse cherche à comprendre pourquoi de nombreux animaux utilisent des signaux coûteux. Par exemple, certaines gazelles sautent verticalement lorsqu'elles aperçoivent un prédateur. Une explication est qu'elles alertent ainsi leur troupeau de la présence hostile. Une autre est qu'elles signalent ainsi au prédateur qu'il a été repéré et qu'elles sont en pleine forme, invitant le prédateur à chercher ailleurs une proie plus facile. De tels signaux existent et ne sont utilisés que s'ils sont crédibles. De nombreux modèles théoriques ont été développé pour «rationaliser» l'usage de signaux par les animaux. En général, les coûts liés au signal sont supposés tels que son usage est «honnête» (c'est-à-dire «séparateur»: les types forts utilisent le signal pour indiquer qu'ils sont forts et les types faibles ne l'utilisent pas). Toutefois, Adams and Caldwell (1990) étudient une population de crustacés (les Gonodactylus bredini) où le signal est souvent utilisé par les types les plus fragiles et où malgré tout il semble crédible, argument formalisé dans Adams and Mesterton-Gibbons (1995). Notre modèle convient parfaitement pour analyser ce genre de situations. L'exemple suivant illustre d'une manière légèrement différente le même phénomène. Les serpents faux corail (très fréquents en Guyane) peuvent être regroupés en deux grandes catégories. Le serpent faux corail peut être très venimeux (par exemple le Erythrolamprus aesculapii de la famille des Colubridae) ou, au contraire, inoffensif (par exemple le Anilius scytale de la famille des Aniliidae). Toutefois, ils sont très difficiles à distinguer par leurs prédateurs. Il en résulte que les serpents inoffensifs sont protégés de manière indirecte par la présence de leurs camarades dangereux. D'une certaine manière les prédateurs sont victimes d'un bluff de la nature.

\section{Conclusion}

Nous avons montré que la présence d'asymétrie d'information perturbe de manière inattendue une situation relativement simple. En effet, si E2 est en information

9. Voir aussi Zahavi and Zahavi (1997). 
parfaite ${ }^{10}$, E1 choisit le grand projet si et seulement si $x>\widetilde{x}$, c'est-à-dire qu'une entreprise qui a un type élevé sélectionne le grand projet, tandis que si elle a un type faible elle opte pour le petit projet. En revanche, lorsque E2 ignore le type de E1, cette séparation bon/mauvais disparaît à l'équilibre. Soit E1 choisit le grand projet quel que soit son type, soit E1 opte pour le grand projet s'il est de type faible ou de type fort mais préfère le petit projet s'il est de type intermédiaire. Il en résulte qu'à l'équilibre, le choix du grand projet signale de manière ambiguë l'habileté de l'entreprise E1.

Une extension possible de notre modèle serait de considérer la situation où la compétition ne conduit pas à un résultat aussi drastique. En particulier, dans certains cas, le gain en cas de compétition dépend non seulement de qui est le plus fort mais aussi de l'écart des forces. Par exemple, si la compétition consiste en une concurrence à la Bertrand (avec $1-x$ le coût marginal de la firme E1 et $1-y$ celui de E2), la firme E1 avec le coût marginal le plus bas $(x>y)$ emporte tout le marché mais sa marge est $y-x$. Il en résulte bien que la taille du gain varie avec l'écart $y-x$. L'intuition est qu'un phénomène de bluff devrait persister dans un tel modèle. Toutefois, il n'est pas forcément réaliste qu'une fois les deux entreprises entrées $x$ et $y$ soient connaissance commune. Si après l'entrée E2 continue d'ignorer $x$ et E1 ne connaît pas $y$, il faudrait alors utiliser les résultats de Spulber (1995) sur la concurrence à la Bertrand avec coûts inconnus.

\section{Références}

Elderidge S. Adams and Roy L. Caldwell. Deceptive communication in asymmetric fights of the stomatopod crustacean gonodactylus bredini. Animal Behavior, 39: 706-713, 1990.

Elderidge S. Adams and Michael Mesterton-Gibbons. The cost of threat displays and the stability of deceptive communication. Journal of Theoretical Biology, 175: 405-421, 1995.

Ken Binmore. Fun and Games. D. C. Health and Company, Lexington, Massachusetts, 1992.

Francis Bloch and Paul Markowitz. Optimal disclosure delay in multistage R \& D competition. International Journal of Industrial Organization, 14(2):159-79, April 1996.

10. C'est-à-dire la situation où E2 connaît le type de E1 avant de choisir d'entrer ou pas mais où E1 ignore le type de E2 avant de sélectionner un projet. 
David Pérez-Castrillo and Thierry Verdier. La structure industrielle dans une course au brevet avec coûts fixes et coûts variables. Revue économique, 42(6):1111-1140, Novembre 1991.

Sylvain Sorin. Bluff et réputation. Revue d'économie politique, 105(4):583-600, Juillet-Août 1995.

Daniel F. Spulber. Bertrand competition when rivals' costs are unknown. Journal of Industrial Economics, 43(1):1-11, March 1995.

Johannes von Neumann and Oskar Morgenstern. Theory of Games and Economic Behavior. Princeton University Press, Princeton, New Jersey, 1944.

Amotz Zahavi. Mate selection - A selection for a handicap. Journal of Theoretical Biology, 53:205-213, 1975.

Amotz Zahavi and Avishag Zahavi. The Handicap Principle. Oxford University Press, Oxford, 1997.

\section{Annexes}

\section{A Preuve de la proposition 3}

Soit $\pi_{1}(x, P)$ l'espérance de profit d'une entreprise E1 de type $x$ qui choisit le petit projet $(\mathrm{P})$. Il est immédiat que

$$
\pi_{1}(x, P)=\int_{0}^{x} g d z-\int_{x}^{1} c d z=(g+c) x-c .
$$

En revanche, l'espérance de gain de $\mathrm{E} 1$, notée $\pi_{1}\left(x, R, \sigma_{2}\right)$, si elle joue $\mathrm{R}$ (gRand projet) dépend de la stratégie $\sigma_{2}($.$) de E2. Il vient:$

$$
\pi_{1}\left(x, R, \sigma_{2}\right)=G \int_{0}^{x} \sigma_{2}(y) d y-C \int_{x}^{1} \sigma_{2}(y) d y+G \int_{0}^{1}\left(1-\sigma_{2}(y)\right) d y
$$

L'expression de cette espérance de gain se simplifie légèrement en

$$
\pi_{1}\left(x, R, \sigma_{2}\right)=G-(G+C) \int_{x}^{1} \sigma_{2}(y) d y .
$$

Étant données ces espérances de gains, la stratégie de E1 est facile à déterminer: si l'espérance de gain est plus élevée avec l'une des deux stratégies, alors il faut 
jouer cette stratégie avec une probabilité égale à 1 , tandis que si les espérances sont identiques E1 est libre de choisir la probabilité avec laquelle il joue R. Formellement:

$$
\sigma_{1}^{*}(x)=\left\{\begin{array}{cl}
0 & \text { si } \pi_{1}\left(x, R, \sigma_{2}\right)<\pi_{1}(x, P) \\
{[0,1]} & \text { si } \pi_{1}\left(x, R, \sigma_{2}\right)=\pi_{1}(x, P) \\
1 & \text { si } \pi_{1}\left(x, R, \sigma_{2}\right)>\pi_{1}(x, P)
\end{array}\right.
$$

Le choix de l'entreprise E2 est le suivant. Soit elle reste en dehors de la compétition et elle a un profit nul. Soit elle entre dans la course et elle obtient $H$ ou $-\gamma$ selon les valeurs de $x$ et de $y$. En espérance elle obtient si elle entre:

$$
\pi_{2}\left(y, E, \sigma_{1}\right)=H \int_{0}^{y} \frac{\sigma_{1}(x)}{\int_{0}^{1} \sigma_{1}(x) d x} d x-\gamma \int_{y}^{1} \frac{\sigma_{1}(x)}{\int_{0}^{1} \sigma_{1}(x) d x} d x
$$

En effet, il faut tenir compte (révision bayésienne) du fait que $x$ n'est plus distribué entre 0 et 1 selon une loi uniforme mais (compte tenu que E1 a joué R) selon la densité $\frac{\sigma_{1}(x)}{\int_{0}^{1} \sigma_{1}(x) d x}$. Après simplification cela conduit à :

$$
\pi_{2}\left(y, E, \sigma_{1}\right)=H-\frac{H+\gamma}{\int_{0}^{1} \sigma_{1}(x) d x} \int_{y}^{1} \sigma_{1}(x) d x
$$

Le joueur 2 entre si cela lui assure une espérance de gain positive. Formellement:

$$
\sigma_{2}^{*}(y)=\left\{\begin{array}{cl}
0 & \text { si } \pi_{2}\left(y, E, \sigma_{1}\right)<0 \\
{[0,1]} & \text { si } \pi_{2}\left(y, E, \sigma_{1}\right)=0 \\
1 & \text { si } \pi_{2}\left(y, E, \sigma_{1}\right)>0
\end{array}\right.
$$

Il est facile de remarquer que la dérivée de $\pi_{2}\left(y, E, \sigma_{1}\right)$ par rapport à y s'écrit

$$
\frac{\partial \pi_{2}\left(y, E, \sigma_{1}\right)}{\partial y}=\frac{H+\gamma}{\int_{0}^{1} \sigma_{1}(x) d x} \sigma_{1}(y) \geq 0
$$

il en résulte donc que la fonction $\pi_{2}\left(y, E, \sigma_{1}\right)$ est croissante (au sens large) avec $y$. De plus, il est facile de vérifier que:

$$
\pi_{2}\left(0, E, \sigma_{1}\right)=-\gamma<0, \text { et } \pi_{2}\left(1, E, \sigma_{1}\right)=H>0
$$

Il existe donc (d'après le théorème des valeurs intermédiaires) $0<y_{0}<1$ et $0<$ $y_{1}<1, y_{0} \leq y_{1}$ tels que $\pi_{2}\left(y, E, \sigma_{1}\right)<0$ pour $0 \leq y<y_{0}, \pi_{2}\left(y, E, \sigma_{1}\right)=0$ pour $y_{0} \leq y \leq y_{1}$, et $\pi_{2}\left(y, E, \sigma_{1}\right)>0$ pour $y_{1}<y \leq 1$. La stratégie optimale de E2 peut donc s'exprimer en termes de ces valeurs $y_{0}$ et $y_{1}$ :

$$
\sigma_{2}^{*}(y)=\left\{\begin{array}{cc}
0 & \text { si } 0 \leq y<y_{0} \\
{[0,1]} & \text { si } y_{0} \leq y \leq y_{1} \\
1 & \text { si } y_{1}<y \leq 1
\end{array}\right.
$$


En utilisant à nouveau le fait que $\frac{\partial \pi_{2}\left(y, E, \sigma_{1}^{*}\right)}{\partial y}=\frac{H+\gamma}{\int_{0}^{1} \sigma_{1}^{*}(x) d x} \sigma_{1}^{*}(y)$, et en utilisant le fait que (par définition de $y_{0}$ et $\left.y_{1}\right)$ pour tout $y, y_{0} \leq y \leq y_{1}, \pi_{2}\left(y, E, \sigma_{1}^{*}\right)=0$, il vient que

$$
\forall y, y_{0} \leq y \leq y_{1}, \frac{\partial \pi_{2}\left(y, E, \sigma_{1}^{*}\right)}{\partial y}=\frac{H+C}{\int_{0}^{1} \sigma_{1}^{*}(x) d x} \sigma_{1}^{*}(y)=0 \Rightarrow \sigma_{1}^{*}(y)=0 .
$$

En outre,

$$
\forall x, x<y_{0}, \pi_{1}\left(x, R, \sigma_{2}^{*}\right)=G+-(G+C) \int_{x}^{1} \sigma_{2}^{*}(y) d y
$$

se simplifie en

$$
G-(G+C) \int_{y_{0}}^{1} \sigma_{2}^{*}(y) d y
$$

et donc

$$
\forall x, x<y_{0}, \pi_{1}\left(x, R, \sigma_{2}^{*}\right) \text { est constant. }
$$

Par définition, $y_{0}$ est la plus petite valeur de $y$ telle que la dérivée de $\pi_{2}\left(y, E, \sigma_{1}^{*}\right)$ s'annule. On a donc qu'en $y_{0}^{-},\left.\frac{\partial \pi_{2}\left(y, E, \sigma_{1}^{*}\right)}{\partial y}\right|_{y=y_{0}^{-}}>0$. C'est-à-dire que $\sigma_{1}^{*}\left(y_{0}^{-}\right)>0$. Or cela n'est possible que si $\pi_{1}\left(y_{0}^{-}, R, \sigma_{2}\right) \geq \pi_{1}\left(y_{0}^{-}, P\right)$. Mais alors, pour tout $x<y_{0}^{-}$, il vient que :

$$
\pi_{1}\left(x, R, \sigma_{2}^{*}\right)=\pi_{1}\left(y_{0}^{-}, R, \sigma_{2}^{*}\right) \geq \pi_{1}\left(y_{0}^{-}, P\right)>\pi_{1}(x, P)
$$

et donc que

$$
\forall x, x<y_{0}, \quad \sigma_{1}^{*}(x)=1 .
$$

D'autre part, pour tout $x>y_{1}$, il vient

$$
\pi_{1}\left(x, R, \sigma_{2}^{*}\right)=G-(G+C) \int_{x}^{1} d y
$$

Il s'agit donc d'une fonction affine, strictement croissante avec $x$ de pente $G+C$. Par ailleurs, l'espérance de gain $\pi_{1}(x, P)$ est aussi une fonction affine, strictement croissante avec $x$ mais de pente $g+c$. Or $g+c<G+C$, il en résulte donc que

$$
\left(\pi_{1}\left(y_{1}^{+}, R, \sigma_{2}^{*}\right) \geq \pi_{1}\left(y_{1}^{+}, P\right)\right) \Rightarrow\left(\pi_{1}\left(x, R, \sigma_{2}^{*}\right)>\pi_{1}(x, P)\right)
$$

et donc que

$$
\forall x, x>y_{1}, \quad \sigma_{1}^{*}(x)=1 .
$$

La stratégie d'équilibre de E1 est donc entièrement déterminée (évidemment, elle dépend des valeurs de $y_{0}$ et de $y_{1}$ qui ne sont toujours pas déterminées. On remarque 
aussi que le cas $y_{0}=y_{1}$ est bien défini, il correspond au cas où E1 choisit toujours le grand projet) :

$$
\sigma_{1}^{*}(x)=\left\{\begin{array}{cc}
1 & \text { si } 0 \leq x<y_{0} \\
0 & \text { si } y_{0} \leq x \leq y_{1} \\
1 & \text { si } y_{1}<x \leq 1
\end{array}\right.
$$

Pour terminer, il faut caractériser la stratégie de E2 de trouver les valeurs de $y_{0}$ et de $y_{1}$ et de déterminer plus précisément $\sigma_{2}^{*}(y)$ pour $y$ compris entre $y_{0}$ et $y_{1}$. Pour cela, on remarque que étant donné $\sigma_{1}^{*}$, il vient tout d'abord que

$$
\int_{0}^{1} \sigma_{1}(x) d x=1-y_{1}+y_{0}
$$

et ensuite que

$$
\pi_{2}\left(y, E, \sigma_{1}^{*}\right)=\left\{\begin{array}{cc}
H-(H+\gamma) \frac{1-y_{1}+y_{0}-y}{1-y_{1}+y_{0}} & \text { si } 0 \leq y<y_{0} \\
H-(H+\gamma) \frac{1-y_{1}}{1-y_{1}+y_{0}} & \text { si } y_{0} \leq y \leq y_{1} \\
H-(H+\gamma) \frac{1-y}{1-y_{1}+y_{0}} & \text { si } y_{1}<y \leq 1
\end{array}\right.
$$

Pour finir, il faut distinguer deux cas. Soit $y_{0}=y_{1}$ et on retrouve le résultat de la proposition 1. Soit $y_{0}<y_{1}$ et alors pour tout $y$ compris entre $y_{0}$ et $y_{1}$, on a par définition de $y_{0}$ et de $y_{1}$ que $\pi_{2}\left(y, E, \sigma_{1}^{*}\right)=0$. Il en résulte que

$$
H-(H+\gamma) \frac{1-y_{1}}{1-y_{1}+y_{0}}=0 \text { soit } H y_{0}+\gamma y_{1}=\gamma
$$

De plus, soit

$$
I=\int_{y_{0}}^{y_{1}} \sigma_{2}^{*}(y) d y
$$

On a pour $x=y_{0}$ et pour $x=y_{1}$ que l'entreprise E1 réalise le même profit quelque soit le projet qu'elle sélectionne d'où d'une part

$$
\pi_{1}\left(y_{0}, R, \sigma_{2}^{*}\right)=\pi_{1}\left(y_{0}, P\right) \Leftrightarrow(g+c) y_{0}-c=(G+C) y_{1}-C-(G+C) I
$$

D'autre part,

$$
\pi_{1}\left(y_{1}, R, \sigma_{2}^{*}\right)=\pi_{1}\left(y_{1}, P\right) \Leftrightarrow(g+c) y_{1}-c=(G+C) y_{1}-C
$$


soit un système de trois équations à trois inconnues à résoudre qui conduit à :

$$
\begin{aligned}
y_{0} & =\frac{\gamma}{H} \frac{G-g}{(G-g)+(C-c)}=\frac{1-\widetilde{x}}{1-\widetilde{y}} \widetilde{y} \\
y_{1} & =\frac{C-c}{(G-g)+(C-c)}=\widetilde{x} \\
\int_{y_{0}}^{y_{1}} \sigma_{2}^{*}(y) d y & =\frac{g+G}{c+C} \frac{H(C-c)-\gamma(G-g)}{H((G-g)+(C-c))}
\end{aligned}
$$

Sous la condition, $\widetilde{x}>\widetilde{y}$, il est immédiat que $y_{0}<y_{1}$. 